\title{
Institutionelle und rechtliche Aspekte \\ der Anpassung ukrainischer Rechtsvorschriften \\ an das Unionsrecht
}

Dr. Roman Petrov

Arthur Weilert

I. Einführung

II. Rechtsgrundlagen der Partnerschaft EU-Ukraine

1. Partnerschafts- und Kooperationsabkommen

2. Europäische Nachbarschaftspolitik und der Aktionsplan EU-Ukraine

3. Assoziierungsabkommen und -agenda

III. Der Umfang des von der Ukraine im Rahmen des PKA anzunehmenden Unionsrechts

IV. Rechtsgrundlagen des Anpassungsprozesses in der Ukraine

V. Institutionelle Mechanismen der Rechtsangleichung in der Ukraine

1. Gemeinsame Institutionen der EU und der Ukraine

2. Anpassungsprozess innerhalb der ukrainischen Exekutive

3. Anpassungsprozess innerhalb der ukrainischen Legislative

4. Anpassungsprozess innerhalb der ukrainischen Jurisdiktion

VI. Schlussbetrachtung

\section{Einführung}

Die Europäisierung der ukrainischen Rechtsordnung begann kurz nach der Erlangung der Unabhängigkeit im Jahre 1991. Anfänglich setzte sich die Ukraine das politische Ziel der Integration in internationale politische sowie ökonomische Strukturen und damit des Beitritts in den Europarat (Council of Europe). Nachdem der Europarat die Kriterien für das Erreichen dieses Ziels festgesetzt hatte, wurden erste Versuche unternommen, eine unionsrechtskonforme Gesetzgebung in Bereichen der Demokratie und der Menschenrechte zu sichern. Dies führte zu grundlegenden Änderungen der Straf-, Strafvollzugs- und Sozialgesetzgebung, etwa durch die Abschaffung der Todesstrafe und die Verabschiedung eines neuen Strafvollzugsgesetzes sowie eines neuen Straf- und Zivilprozessrechts. Die ersten Schritte in Richtung der Aufnahme „europäischer Rechtsnormen“ in die sich sichtbar entwickelnde ukrainische Rechtsordnung waren somit getan.

Die Entscheidung der Ukraine, einen neuen politischen Kurs einzuschlagen, der eine Annäherung an die EU vorsieht, wurde 1994 mit der Unterzeichnung eines Part- 
nerschafts- und Kooperationsabkommens ${ }^{1}$ (PKA) mit der Europäischen Gemeinschaft (EG) und ihren Mitgliedstaaten manifestiert. Teil des PKA ist die „soft law Verpflichtung“ der Ukraine, „sich darum zu bemühen, dass ihre Rechtsvorschriften schrittweise mit dem Gemeinschaftsrecht vereinbar werden" (Art. 51 Abs. 1 S. 2 PKA EU-Ukraine2). Insoweit kann von einer "Angleichungsklausel“ gesprochen werden. In deren Ausgestaltung hat die Ukraine den Gedanken der "Anpassung“ des innerstaatlichen Rechts an das Unionsrecht entworfen. ${ }^{3}$

Dieser Beitrag legt den Fokus auf verschiedene Faktoren der Anpassung ukrainischer Rechtsvorschriften an das Unionsrecht. Hierfür werden zunächst die rechtlichen Grundlagen der Partnerschaft zwischen der EU und der Ukraine sowie deren Einfluss auf ihre zukünftige Beziehung dargelegt (II.). Anschließend werden Inhalt und Natur des Anpassungsprozesses in der Ukraine betrachtet (III. und IV.). Ferner behandelt der Beitrag die institutionellen Mechanismen der Anpassung, insbesondere unter Berücksichtigung des Einflusses des nationalen Programms der Anpassung auf die ukrainische Jurisdiktion (V.). Zuletzt wird eine Schlussfolgerung hinsichtlich des jüngsten Stands der Angleichung des ukrainischen Rechts und ihren zukünftigen Herausforderungen angestellt (VI.).

\section{Rechtsgrundlagen der Partnerschaft EU-Ukraine}

\section{Partnerschafts- und Kooperationsabkommen}

Das rechtliche Rahmenwerk, das die Beziehungen zwischen der EU und der Ukraine regelt, gipfelte in dem von der EG und ihren Mitgliedstaaten einerseits und der Ukraine andererseits am 16. Juni 1994 unterzeichneten und am 1. März 1998 in Kraft getretenen PKA. ${ }^{4}$ Dieses Abkommen war für einen Zeitraum von zehn Jahren ge-

\footnotetext{
1 Abkommen über Partnerschaft und Zusammenarbeit zwischen den Europäischen Gemeinschaften und ihren Mitgliedstaaten und der Ukraine, ABl. L 49 vom 19. Februar 1998, S. 3 - 46.

${ }^{2}$ Im Folgenden zitierte Artikel eines PKA ohne nähere Bezeichnung sind Artikel des PKA EU-Ukraine.

${ }^{3}$ Grundsätzlich zur Rechtsangleichung siehe Gialdino, Some Reflections on the Acquis Communautaire, Common Market Law Review 1995, S. 1089 - 1121; Maresceau/Montaguti, The Relations between the European Union and Central and Eastern Europe: A Legal Appraisal, Common Market Law Revier 1995, S: 1327 - 1367; Delcourt, The Acquis Communautaire: Has The Concept had its Day?, Common Market Law Review 2001, S. 829 - 870; Weatherill, Safeguarding the acquis communautaire, in: Heukels/Blokker/Brus (Hrsg.), The European Union after Amsterdam - a legal Analysis (Den Haag, 1998), S. 153 - 178, 161 f.; Goebel, The European Union Grows: The Constitutional Impact of The Accession of Austria, Finland and Sweden, Fordham International Law Journal 1995, S. 1092 - 1190 , 1141 f.; Hillion, Approximation of laws in the context of EU-NIS partnership, in: Ficzere/Kellermann/Nikodem (Hrsg.), Perspectives of the legal Approximation Process in Cetral and Eastern Europe (Budapest, 2001), S. 78 - 86; Muraviov, Pravovi zasady regulyvania ekonomichikh vidnosyn Evropeiskogo Souzy z tretimy krainamy - teoria I praktika (Kiew, 2002); Cremona, State Aid Control: Substance and Procedure in the Europe Agreements and the Stabilisation and Association Agreements, European Law Journal 2003, S. 265 - 287.

4 Entsprechende PKA wurden unterzeichnet mit Armenien, Aserbaidschan, Belarus (trat nicht in Kraft), Georgien, Kasachstan, Kirgistan, Russland, Usbekistan, Turkmenistan (noch nicht in Kraft getreten).
} 
schlossen worden. Seit Anfang 2008 verlängert es sich automatisch für ein Jahr, sofern nicht eine Partei das Abkommen kündigt (Art. 101 PKA).

Als völkerrechtlicher Vertrag (erweitertes Handelsabkommen) ${ }^{5}$ entfaltet das PKA EU-Ukraine bindende Wirkung und begründet einen integrierenden Bestandteil der Unionsrechtsordnung sowie der Rechtsordnungen der Mitgliedstaaten. ${ }^{6}$ Darüber hinaus haben zumindest einige Bestimmungen des PKA potenziellen Vorrang gegenüber kollidierenden europarechtlichen Regelungsnormen und nationalen Rechtsvorschriften der Mitgliedstaaten. ${ }^{7}$ In der ukrainischen Rechtsordnung hat das PKA bindende Wirkung und genießt folglich Priorität gegenüber jeglicher konkurrierender Nationalgesetzgebung, obgleich es nicht über der Ukrainischen Verfassung steht. Im Falle einer verfassungsrechtlichen Kollision setzt sich daher die ukrainische Verfassungsbestimmung entweder durch oder sie muss geändert werden. ${ }^{8}$

Das PKA EU-Ukraine kann (wie auch andere PKA) als „Einstiegs-Abkommen“ klassifiziert werden, das zwar keine Mitgliedschaft vorsieht, aber das Interesse an der Entwicklung weiterer beiderseitiger Zusammenarbeit der Parteien bestätigt. Hauptsächlich zielt es ab auf die Errichtung eines politischen Dialogs, die Förderung von Wirtschaftsbeziehungen und demokratischen Reformen in der Ukraine, den Menschenrechtsschutz und die Errichtung einer Rechtsordnung, die Rechtsstaatlichkeit garantiert. Die Ausführungen der Präambel des PKA EUUkraine vermeiden jede Bezugnahme auf einen „Europäischen Integrationsprozess" oder das "Ziel einer EU-Mitgliedschaft", wie es in den EU-Assoziierungsabkommen mit mittel- und osteuropäischen Ländern statuiert wurde. Vorerst erfüllt das PKA mit Sicherheit seinen Zweck als verlässliches Rechtsinstrument zur Stützung der langfristigen Beziehungen zur Ukraine, während es sie auf kontrollierbarer Distanz zu einem näheren Zugang zum EU-Binnenmarkt hält. ${ }^{9}$ Aus Sicht der EUAußenpolitik kann es somit als Erfolgsformel angesehen werden.

5 Lankosz, Die Partnerschaftsabkommen mit osteuropäischen Nachbarstaaten, in: Lankosz/MüllerGraff/Fink (Hrsg.), Osteuropäische Nachbarschaft der Europäischen Union (Baden-Baden, 2009), S. 15-20, 15.

${ }^{6}$ Zur Anerkennung der Bestimmungen eines internationalen Abkommens als integrierender Bestandteil der Gemeinschaftsrechtsordnung durch den EuGH siehe EuGH, Urteil vom 26. Oktober 1982, Rs. C 104/81, Kupferberg, Slg. 1982, S. 3641, Rn. 13.

${ }^{7}$ Die Rechtsprechung des EuGH fordert die Erfüllung zweier Bedingungen, bevor ein Rechtsakt der EU aufgrund seiner Unvereinbarkeit mit einem internationalen Abkommen für unwirksam erklärt werden kann. Erstens muss die EU durch die Bestimmung des betreffenden internationalen Abkommens gebunden werden. Zweitens muss diese Bestimmung eine unmittelbar anwendbare unionsrechtliche Vorschrift sein. Zu gemischten Abkommen in der Rechtsordnung der EU und den Rechtsordnungen der Mitgliedstaaten siehe EuGH, Urteil vom 30. September 1987, Rs. C 12/86, Demirel, Slg. 1987, S. 3719. Hinsichtlich einer möglichen unmittelbaren Anwendbarkeit von PKA siehe Petrov, Rights of Third Country Nationals/Newly Independent States' Nationals to Pursue Economic Activity in the EU, European Foreign Affairs Review 1999, S. 235-251.

${ }^{8}$ Art. 9 der Ukrainischen Verfassung besagt, dass „,...] internationale und vom Parlament der Ukraine gebilligte völkerrechtliche und in Kraft getretene Vereinbarungen Teil der nationalen Gesetze der Ukraine sind. Der Abschluss internationaler, der Verfassung der Ukraine zuwiderhandelnder Verträge, ist nur nach entsprechender Anpassung der Verfassung der Ukraine möglich."

${ }^{9}$ Zur vergleichenden Übersicht und einer ausführlichen Untersuchung der PKA siehe Petrov, The Partnership and Cooperation Agreements with the Newly Independent States, in: Ott/Inglis (Hrsg.), Handbook on European Enlargement (Den Haag, 2002), S. 175-194. 
Das heutige Niveau der Zusammenarbeit zwischen den Parteien spiegelt das PKA dennoch nicht wieder, wie die Verhandlungen der EU und Ukraine über ein neues vertieftes bilaterales Abkommen (new enhanced agreement), das ein Assoziierungsabkommen auf der Grundlage des Art. 217 des Vertrages über die Arbeitsweise der Europäischen Union (AEUV) sein wird, zeigen. Das Assoziierungsabkommen bedeutet noch keine Vollmitgliedschaft in der EU. ${ }^{10}$ Es wird auf eine verstärkte politische und wirtschaftliche Zusammenarbeit einschließlich der Errichtung einer ausnahmslosen Freihandelszone zwischen den Parteien gerichtet sein. Die EU ist jedoch aufgrund der unruhigen politischen Situation in der Ukraine nicht gerade bestrebt, den Verhandlungsprozess zu beschleunigen. Das PKA bleibt damit ein bedeutender bilateraler Rahmenvertrag, um die EU-Ukraine-Beziehungen in naher Zukunft zu regeln. ${ }^{11}$

\section{Europäische Nachbarschaftspolitik und der Aktionsplan EU-Ukraine}

Am Vorabend der beispiellosen Erweiterung der EU in Richtung Mittel- und Osteuropa im Jahre 2004 leitete die Kommission die "Europäische Nachbarschaftspolitik“ (ENP) zu jenen Drittländern ein, die nach der Erweiterung unmittelbar an die EU grenzten. ${ }^{12}$ Neben anderen Nachbarländern ernannte die Kommission die Ukraine zum „privilegierten Partner" und verkündete öffentlich folgende Ziele: Die Errichtung einer EU-Ukraine-Freihandelszone und der Zugang zu ausgewählten Teilen des EU-Binnenmarktes sowie „Finanzpaketen“. Im Gegenzug bestärkt die ENP die Ukraine - trotz fehlender Beteiligung an der EU-Entscheidungsfindung und dem Rechtsetzungsverfahren - ihr System zu transformieren und die freiwillige Übernahme des acquis communnautaire (gemeinschaftlicher Besitzstand) fortzusetzen.

Die Unterzeichnung des bilateralen Aktionsplans durch die Kommission und die ukrainische Regierung im Februar 2005 stellte den Beginn einer neuen Etappe in den Beziehungen der EU zur Ukraine dar. Aktionspläne kennzeichneten nach der Schaffung der ENP den Charakter der bilateralen Beziehungen zwischen der Union und ihren Nachbarländern für eine dreijährige Gültigkeit. Folglich waren die Aktionspläne nicht identisch. Sie wurden geschlossen, um den individuellen Bedürfnis-

10 Tiede/Schirmer, Die Östliche Partnerschaft der Europäischen Union im Rahmen des Gemeinschaftsrechts, Osteuropa-Recht 2009, S. 184-191, 187. In dem möglichen Abschluss des Abkommens immerhin eine realistische Chance auf einen EU-Beitritt sehend Tiede/Krispenz/Schirmer, Aktuelle Entwicklungen im ukrainischen Justizwesen einschließlich europarechtlicher und völkerrechtlicher Bezüge, WiRO 2010, S. 33-38 (Teil 1), S. 75-80 (Teil 2), 75.

11 Ausführlich zur Thematik eines zukünftigen Assoziierungsabkommens zwischen der EU und der Ukraine siehe Petrov, Legal basis and scope of the new EU-Ukraine enhanced agreement. Is there any room for further speculation?, European University Institute Working Papers (Max Weber Programme), 2008/17.

12 Mitteilung der Kommission an den Rat und das Europäische Parlament „Größeres Europa - Nachbarschaft: Ein neuer Rahmen für die Beziehungen der EU zu ihren östlichen und südlichen Nachbarn“, KOM (2003) 104 endgültig. Die ENP bezieht sich auf Nachbarländer, die unmittelbar an die EU grenzen: Ukraine, Belarus, Ägypten, Israel, Libanon, Jordanien, Marokko, Moldawien, Palästinensische Autonomiebehörde und Tunesien. Mittlerweile schließt sie auch Länder wie Armenien, Aserbaidschan und Georgien ein, die mit der EU keine unmittelbare Grenze haben. 
sen der Nachbarländer im Zuge der Annäherung an die EU gerecht zu werden. Gleichzeitig enthielten sie einige allgemeine Elemente, die den grundlegenden Zielen der ENP entsprachen, ${ }^{13}$ nämlich das Einhalten allgemeiner demokratischer Werte, die Errichtung einer funktionsfähigen Marktwirtschaft, und die Annäherung der Rechtssysteme der Nachbarländer an das Unionsrecht. Die ENP hält die Nachbarländer an, den acquis communautaire freiwillig zu übernehmen, obgleich diese weder an der EU-Beschlussfassung noch am Rechtsetzungsverfahren beteiligt sind. So dienen bilaterale Aktionspläne vor allem dazu, den genauen Umfang des von dem jeweiligen benachbarten Staat anzunehmenden acquis communautaire zu klären. ${ }^{14}$

Eines der Hauptziele des Aktionsplanes mit der Ukraine war es, vorrangige Bereiche für innerstaatliche Reformen zu skizzieren. Im Vergleich zu den übrigen Aktionsplänen wurde die freiwillige Harmonisierung im Aktionsplan EU-Ukraine am stärksten betont. So bekräftigte dieser die Ukraine, rechtsetzende und institutionelle Grundlagen weiterzuentwickeln, um schließlich nicht den gesamten, aber doch einen beachtlichen Teil des acquis communautaire zu übernehmen. Folgende Bereiche, in denen von der ukrainischen Regierung innerhalb der dreijährigen Frist gesetzliche Reformen durchzuführen waren, wurden erfasst: die Übernahme und Durchsetzung von gemeinsamen europäischen Werten (Demokratie, Rechtsstaatlichkeit, Menschenrechte und Grundfreiheiten), das Festhalten an grundlegenden demokratischen Prinzipien (Achtung der Pressefreiheit sowie der Rechte nationaler Minderheiten, Schutz der Rechte von Kindern), die Unterzeichnung und Durchsetzung internationaler Konventionen (Statut des Internationalen Strafgerichtshofs [ICC], Resolutionen des UN-Sicherheitsrates), die Teilnahme an internationalen Initiativen für Demokratie und Sicherheit (Nichtverbreitung von Massenvernichtungswaffen, Bekämpfung des Terrorismus) und die Zusammenarbeit in der EU Außen- und Sicherheitspolitik. Das Ziel des Aktionsplans, die Implementierung und Durchsetzung des beträchtlichen Umfangs an völkerrechtlichen Dokumenten durch die Ukraine sicherzustellen, hatte die Revision jener Bestimmungen der Ukrainischen Verfassung zur Folge, die den Vorrang des Völkerrechts innerhalb der ukrainischen Rechtsordnung begründen konnten. Im Bereich der institutionellen Reformen wurde die Ukraine aufgefordert, ein Ministerium für Europäische Integration zu gründen, das dazu fähig ist, den Angleichungsprozess innerhalb der Exekutive, Legislative und Jurisdiktion zu koordinieren.

${ }^{13}$ Beichelt, Externe Demokratisierungsstrategien der Europäischen Union: Die Fälle Belarus, Moldava, Ukraine, in: Jünemann/Knodt (Hrsg.), Externe Demokratieförderung durch die Europäische Union (2007), S. 207-230, 219. Angesichts der Erwartung, der Aktionsplan werde präziser sowie verbindlicher als das bestehende PKA EU-Ukraine, war die Kritik bei seinem Abschluss nicht zu überhören, die neben höherer Präzision vor allem entsprechende Integrationsanreize und Unterstützung in Form von Gegenleistungen seitens der EU forderte, vgl. nur Stratenschulte, Ukraine: „Und jetzt: action!“, Osteuropa 2005, S. 15-23, 17 ff. („,Der Aktionsplan ist nicht mehr, als weiße Salbe auf der Wunde der Zurückweisung des Beitrittswunsches“, S. 20); Böttger, Die Entstehung und Entwicklung der Europäischen Nachbarschaftspolitik (2010), S. 99 ff.; Koopmann, Die Nachbarschaftspolitik der Europäischen Union: Herausforderungen und Probleme eines anspruchsvollen Politikkonzepts, in: Koopmann/Lequesne, Partner oder Beitrittskandidaten? (2006), S. 17-30, 28 ff.

${ }^{14}$ Neuere Informationen über die Aktionspläne und die Assoziierungsagenda EU-Ukraine im Rahmen der ENP verfügbar unter: http://ec.europa.eu/world/enp/documents_en.htm, letzter Zugriff am 2. November 2010. Abgesehen von Belarus wurden Aktionspläne mit allen ENP-Ländern geschlossen. 


\section{Assoziierungsabkommen und -agenda}

Der Aktionsplan EU-Ukraine wurde für die Dauer von drei Jahren geschlossen und lief daher Anfang des Jahres 2008 ab - zur gleichen Zeit, zu der auch das PKA EUUkraine endete. Wie erwähnt, wurden im März 2007 Verhandlungen über ein Assoziierungsabkommen in die Wege geleitet, um das überholte PKA zu ersetzen. Aufgrund andauernder politischer Instabilität in der Ukraine ist ein Ende des Verhandlungsprozesses jedoch nicht abzusehen. Bis dahin wird das PKA jährlich um weitere zwölf Monate verlängert. Für den Aktionsplan kam eine entsprechende temporäre Lösung hingegen nicht in Betracht. Um die „europäischen Ambitionen“ der Ukraine aufrechtzuerhalten wurde dieser am 23. November 2009 auf dem alljährlichen Gipfeltreffen der EU und der Ukraine durch die neue Assoziierungsagenda EU-Ukraine abgelöst. Dabei fußt die neue Assoziierungsagenda fest auf dem Boden des Aktionsplans. Sie ist ein umfassendes Rahmendokument, dazu berufen, Demokratie, Wirtschaftsreformen und Rechtsstaatlichkeit zu fördern. Aufgabe der Assoziierungsagenda ist es damit, notwendige Ziele für innerstaatliche Reformen zu begründen, bis das Assoziierungsabkommen in Kraft tritt. Hinsichtlich der Angleichung ukrainischer Rechtsvorschriften an das Unionsrecht verfolgt die Assoziierungsagenda weniger ehrgeizige Ziele als der Aktionsplan EU-Ukraine. Statt einer horizontalen Annäherung im Sinne des Aktionsplans arbeitet die Assoziierungsagenda mit einer sektoralen Herangehensweise. Die Ukraine wird angehalten, die "progressive Annäherung" nationaler Rechtsvorschriften an konkrete Bereiche des Unionsrechts (u.a. Gesundheitswesen, Besteuerung, Gesellschaftsrecht) fortzusetzen, was mit Vereinheitlichung, Konformitätsbewertung und regelungstechnischer Umsetzung verbunden ist. In anderen Bereichen der sektoralen Zusammenarbeit (etwa Wettbewerbsrecht, Zollrecht, Energie, Buchführung und Rechnungswesen) bemüht sich die Ukraine, standardisierte Verfahren der EU zu übernehmen. Vor diesem Hintergrund ist festzustellen, dass die Assoziierungsagenda das Ziel einer eher zaghaften Angleichung der ukrainischen Rechtsvorschriften an den acquis communautaire verfolgt und dabei von einer großen Reichweite des Ermessens ukrainischer Behörden ausgeht.

\section{Der Umfang des von der Ukraine im Rahmen des PKA anzunehmenden Uni- onsrechts}

Der Umfang des Unionsrechts, der als Modell für die Angleichung herangezogen werden kann, ist eher fragmentiert. Die "Angleichungsklausel“ des Art. 51 Abs. 1 S. 2 PKA statuiert zunächst die „soft law - Verpflichtung“ der Ukraine, „sich darum zu bemühen, dass ihre Rechtsvorschriften schrittweise mit dem Gemeinschaftsrecht vereinbar werden“. Art. 51 Abs. 2 PKA listet daneben bestimmte Bereiche auf, die vom Angleichungsprozess in der Ukraine vorrangig erfasst werden sollen. ${ }^{15}$ Inner-

15 Diese sind: Zollrecht, Gesellschaftsrecht, Bankenrecht, Rechnungslegung der Unternehmen und Steuern, geistiges Eigentum, Schutz der Arbeitnehmer am Arbeitsplatz, Finanzdienstleistungen, 
halb des PKA EU-Ukraine vollzieht sich hier der Wandel von sonst die Außenbeziehungen betreffenden Vorschriften hin zu der Verpflichtung, ukrainisches Recht im Einklang mit dem Unionsrecht zu modifizieren. ${ }^{16}$ Dieser Aspekt verdeutlicht wiederum die "gewisse Asymmetrie“ der korrelierenden Verpflichtungen, die sich durch alle PKA zieht und darin äußert, dass sich der Partnerstaat zur Verwirklichung der PKA-Ziele zu einem Transformationsprozess verpflichtet. ${ }^{17}$

Darüber hinaus betont das PKA EU-Ukraine die Notwendigkeit der Anwendung bestimmter völkerrechtlicher Regelungen, etwa des Allgemeinen Zoll- und Handelsabkommens (General Agreement on Tariffs and Trade, GATT), und der Implementierung völkerrechtlicher Instrumente. Die Ukraine hat sich weiter dazu verpflichtet, bis zum Jahre 2002 (Ende des fünften Jahres nach Inkrafttreten des PKA) einem umfangreichen Katalog an multilateralen Übereinkünften über die Rechte an geistigem, gewerblichem und kommerziellem Eigentum beizutreten (Art. 50 Abs. 2 PKA). ${ }^{18}$ Art. 2 PKA ruft die Ukraine dazu auf, die „wesentlichen Bestandteile“ der Partnerschaft zu erfüllen. Genannt werden an dieser Stelle die Achtung der Grundsätze der Demokratie und der Menschenrechte sowie die Grundsätze der Marktwirtschaft. Es überrascht nicht, dass der Bereich der „wesentlichen Bestandteile“ im PKA nicht genau definiert wird. Allerdings wird auf völkerrechtliche Konventionen und Dokumente allgemeiner Geltung Bezug genommen, was der EU ein breites politisches Manövrieren ermöglicht. ${ }^{19}$ Sollte gegen die in Art. 2 PKA niedergelegten „wesentlichen Bestandteile“ verstoßen werden, bedeutet dies eine erhebliche Verletzung des Abkommens und stellt einen „besonders dringenden Fall“ nach Art. 102 Abs. 2 S. 2 PKA dar, der dazu berechtigt, die Implementierung des Abkommens unilateral aufzuheben. ${ }^{20}$

Wettbewerbsregeln, öffentliches Auftragswesen, Schutz der Gesundheit und des Lebens von Menschen, Tieren und Pflanzen, Umwelt, Verbraucherschutz, indirekte Steuern, technische Vorschriften und Normen, Gesetze und sonstige Vorschriften für den Nuklearbereich, Verkehr.

16 Schröter, Angleichung an das Recht der EG in Ostmitteleuropa und Osteuropa, in: Lankosz/MüllerGraff/Fink (Fn. 5), S. 69-80, 77.

${ }^{17}$ Lankosz (Fn. 5), S. 15-20, 17.

${ }^{18}$ Eine Auflistung der multilateralen Übereinkünfte findet sich im Anhang III des PKA, auf den auch Art. 50 Abs. 1 und Abs. 2 PKA verweisen.

19 „Wesentlicher Bestandteil“ der Partnerschaft und des Abkommens sind gem. Art. 2 PKA "die Achtung der Grundsätze der Demokratie und der Menschenrechte, wie sie insbesondere in der Schlussakte von Helsinki und in der Pariser Charta für ein neues Europa definiert sind, sowie die Grundsätze der Marktwirtschaft, wie sie unter anderem in den Dokumenten der KSZE-Konferenz in Bonn aufgestellt werden".

${ }^{20}$ Siehe angefügte gemeinsame Erklärungen zu dem PKA EU-Russische Föderation (dort gemeinsame Erklärung zu Art. 107) und dem PKA EU-Ukraine (gemeinsame Erklärung zu Art. 102 PKA). Die EU "wacht“ über die Verwirklichung der „wesentlichen Bestandteile” mittels einer Einzelfallprüfung. So ratifizierte die EU das PKA mit Belarus aufgrund von Verstößen gegen Demokratie, Rechtsstaatlichkeit und Menschenrechte als „wesentliche Bestandteile“ nicht, wohingegen eben diese Gründe im Hinblick auf Menschenrechtsverletzungen in Tschetschenien nicht dieselben Folgen für die Russische Föderation hatten; hieran erkennt man die politische Natur des Entscheidungsprozesses im Rat für Allgemeine Angelegenheiten; siehe Erklärung der EU hinsichtlich der Beziehungen zu Belarus (Tagung des Rates vom 29./30. April 1997), Pressemitteilung der EU, Nr. $7738 / 97$ (129) und Schlussfolgerungen des Rates zu Belarus (Tagung vom 15. September 1997), Pressemitteilung der EU, Nr. $10368 / 97$ (269). 
Zudem besagt die Präambel des PKA EU-Ukraine, dass „eine feste Verpflichtung [...] der Ukraine zur vollen Verwirklichung aller Grundsätze und Bestimmungen der Schlussakte der Konferenz über Sicherheit und Zusammenarbeit in Europa (KSZE), der Abschließenden Dokumente der Folgetreffen in Madrid und Wien, des Dokuments der KSZE-Konferenz in Bonn über wirtschaftliche Zusammenarbeit, der Pariser Charta für ein neues Europa und des Dokuments der KSZE-Konferenz in Helsinki von 1992, 'Herausforderung des Wandels “", besteht.

Nicht die Statuierung vage formulierter, in ihrer Reichweite begrenzter "Angleichungsklauseln“ bildet grundsätzlich den Schwerpunkt innerhalb der PKA, sondern die Anwendung völkerrechtlicher Verträge, wie des GATT, und die Implementierung internationaler Übereinkünfte im Bereich des geistigen Eigentums sowie unverbindlicher KSZE-Dokumente. In diesem Sinne wird die "Angleichungsklausel“ des PKA EU-Ukraine auch nicht als Hauptziel des Abkommens betrachtet. Dies stellt den Vorrang der Angleichung innerhalb bilateraler EU-PKA in Frage, was schlechthin die „soft law - Natur" der Verpflichtung aufzeigt. Der der „Angleichungsklausel“ innewohnende Katalog vorrangiger Bereiche zeigt keine kohärente EU-Leitlinie für die Frage nach dem Umfang und dem Gehalt des zu übernehmenden Unionsrechts. Deshalb sollte die im PKA enthaltene "Angleichungsklausel“ in einem neuen Assoziierungsabkommen zwischen der EU und der Ukraine noch einmal überdacht werden.

\section{Rechtsgrundlagen des Anpassungsprozesses in der Ukraine}

Mit dem Inkrafttreten des PKA am 01. März 1998 wurde die ukrainische Regierung mit ernsthaften Schwierigkeiten konfrontiert, die mit den Methoden und Mitteln der Durchführung und Durchsetzung der PKA-Bestimmungen innerhalb der nationalen Rechtsordnung in Zusammenhang standen. Schon im Jahre 1994 war deutlich absehbar, dass es erheblichen Aufwand bedeuten würde, in der ukrainischen Rechtsordnung, die das Erbe einer sozialistischen Rechtsordnung war, die rechtlichen, institutionellen, wirtschaftlichen und politischen Veränderungen nach den Vorstellungen des PKA zu vollziehen.

Seit 1998 haben der Präsident und die Regierung der Ukraine zwecks der Durchsetzung des PKA eine Reihe von Rechtsakten verabschiedet. ${ }^{21}$ Der allgemeine Rahmen des Integrationsprozesses wurde in der „Strategie der Integration der Ukraine in die $\mathrm{EU}^{\prime \prime 22}$ (Strategie der Integration) errichtet. Dieses Dokument bestimmt die wesentlichen Prioritäten für die Exekutive, die auf das endgültige Ziel, die EU-

\footnotetext{
${ }^{21}$ Inzwischen haben die Werchowna Rada (Parlament) und die Regierung etwa 100 Rechtsakte im Bereich der Integration der Ukraine in die EU verabschiedet.

22 Erlass (Ukas) des Präsidenten der Ukraine „Zur Billigung `der Strategie der Integration der Ukraine in die Europäische Union'“, vom 11. Juni 1998, Nr. 615/98.
} 
Mitgliedschaft schnellstmöglich zu erwerben, gerichtet sind. ${ }^{23}$ An sich proklamierte der Präsident der Ukraine, dass der Beitritt zum Europäischen Politischen, Wirtschafts- und Rechtsraum und der anschließende Erwerb einer assoziierten Mitgliedschaft der EU von vorrangiger Bedeutung für die mittelfristige ukrainische Außenpolitik seien. Kurz darauf wurde der Kompetenzbereich der Exekutivbehörden definiert und der entsprechende institutionelle Rahmen mit dem Zweck geschaffen, den Integrationsprozess zu beschleunigen und das PKA EU-Ukraine zu implementieren. ${ }^{24}$

Von Anfang an hat die Regierung der Ukraine den Prozess der "Anpassung“ nationaler Rechtsvorschriften an die Standards der EU vorangetrieben, wobei sie dies von dem Terminus der "Angleichung“, der grundsätzlich im Beitrittsprozess eines Drittstaates zur EU verwendet wird, unterschied. Die Anpassung ukrainischer Rechtsvorschriften an das Unionsrecht wurde formell im Jahre 1999 aufgenommen, als das Ministerkabinett der Ukraine das „Konzept der Anpassung Ukrainischer Rechtsvorschriften an die Gesetzgebung der EU“25 (Konzept der Anpassung) ausfertigte, in dem das offizielle Verständnis des Anpassungsprozesses niedergelegt wurde. Die allgemeinen Ziele und der Umfang des Anpassungsprozesses in der Ukraine, wie die Angleichung nationaler Rechtsvorschriften an das gegenwärtige Europäische Rechtssystem, wurden bereits weitgehend in der Strategie der Integration definiert. ${ }^{26}$ Gemäß der Strategie der Integration wurde der Anpassungsprozess in der Ukraine darauf ausgerichtet, das PKA zu implementieren, sektorale Abkommen mit der EU abzuschließen sowie ukrainische Rechtsvorschriften zu formen und näher an das Unionsrecht zu bringen.

Kurz nachdem das Konzept der Anpassung in Kraft trat, erließ der Präsident der Ukraine das umfassende „Programm der Integration in die EU“27 (Programm der Integration), das einen Rahmen von kurz-, mittel- und langfristigen Zielen für die Exekutive darstellt, um die Ukraine in die EU zu integrieren. Darin wurden die Kopenhagener Kriterien als Voraussetzung für die Mitgliedschaft in der EU ausdrücklich befürwortet und als grundlegende Rahmenbedingungen für die Integrationsbemühungen in der Ukraine akzeptiert. Das Programm der Integration festigt die konkreten

\footnotetext{
${ }^{23}$ Die ursprüngliche Frist, um sich bis 2007 für die volle Mitgliedschaft zu qualifizieren, wurde jüngst bis 2011 verlängert. Die Frist zum Erwerb einer WTO-Mitgliedschaft wurde 2003 aufgestellt. Apell des Präsidenten der Ukraine an die Werchowna Rada der Ukraine „Europäische Wahl. Begriffsgrundlagen der Strategie der wirtschaftlichen und sozialen Entwicklung der Ukraine in den Jahren 2002 2011“, vom 20. Juni 2002, Nr. 20-IV.

${ }^{24}$ Anordnung (Rasporiadzhenie) des Präsidenten der Ukraine „Über die Liste der für die Erfüllung der von der 'Strategie der Integration der Ukraine in die EU' definierten Aufgaben verantwortlichen Regierungsstellen“, vom 27. Juni 1999, Nr. 151/99-rp (geändert durch Erlass des Präsidenten der Ukraine, am 06. Juli 2000, Nr. 240/2000).

${ }^{25}$ Verordnung des Ministerkabinetts der Ukraine „Konzept der Anpassung der Gesetzgebung der Ukraine an die Gesetzgebung der EU“, vom 16. August 1999, Nr. 1496. Jüngst scheinen von der Regierung der Ukraine erlassene Rechtsakte gleichzeitig und manchmal austauschbar Definitionen von "Anpassung“, „Annäherung“ und „Harmonisierung“ zu verwenden, ohne den Unterschied ihres Sinngehalts zu klären.

${ }^{26}$ Art. 1 des Erlasses des Präsidenten der Ukraine „Zur Billigung `der Strategie der Integration der Ukraine in die Europäische Union ““, vom 11. Juni 1998, Nr. 615/98.

27 „Programm der Integration in die Europäische Union“, genehmigt durch Erlass des Präsidenten der Ukraine, vom 14. September 2000, Nr. 1072/2000.
} 
Ziele politischer, wirtschaftlicher und rechtlicher Reformen durch eine sorgfältige Analyse des gegenwärtigen Stands der Demokratie und der Rechtsstaatlichkeit, der Reform von Verwaltung und Justiz, des Schutzes der Menschenrechte und der Wirtschaftsentwicklung in der Ukraine. Obwohl sich das Programm der Integration sowohl als deklaratives wie auch normatives Dokument mit detaillierten Leitlinien erwies, ist sein praktischer Einfluss auf das Ankurbeln neuer Maßnahmen zum Zwecke der Integration der Ukraine in die EU beschränkt.

Um die Ziele des Programms der Integration zu erreichen, erlässt das Ministerkabinett jährliche Anpassungs-Aktionspläne, die einen bestimmten Katalog von organisatorischen und gesetzgeberischen Maßnahmen aufstellen, welche ihrerseits im Laufe des Kalenderjahres durchzuführen und zu verabschieden sind. Auf der Zusammenarbeit mit internationalen Institutionen und der Durchsetzung völkerrechtlicher Konventionen innerhalb der Ziele des Programms der Integration liegt das Hauptaugenmerk der jährlichen Anpassungs-Aktionspläne. Als Gegenpart zu dem Aktionsplan des Ministerkabinetts erlassen alle Ministerien und Regierungsbehörden, die am Integrationsprozess der Ukraine in die EU beteiligt sind, eigene jährliche AnpassungsAktionspläne.

Das wichtigste Rahmengesetz über die Anpassung ukrainischen Rechts an den $a c$ quis communautaire wurde im Jahre 2004 erlassen (Programm der Anpassung). ${ }^{28}$ Das Programm der Anpassung sieht verschiedene Stufen des Anpassungsprozesses vor. Innerhalb der ersten Stufe (2004 - 2007) verpflichtet sich die Ukraine, ihre Rechtsvorschriften in den in Art. 51 Abs. 2 PKA aufgelisteten vorrangigen Bereichen an den acquis communautaire anzugleichen. Umfang und Dauer der folgenden Stufen werden aufgrund der Verbindung zum Niveau und Fortschritt der Beziehungen zwischen der EU und der Ukraine nicht angegeben. Jedoch strebt das Programm der Anpassung den allmählichen Import des gesamten „acquis“ in die Rechtsordnung der Ukraine an, da das Ziel dieses Gesetzes die „Angleichung ukrainischer Rechtsvorschriften an den acquis communautaire, unter Berücksichtigung der von EU gegenüber beitrittswilligen Staaten spezifizierten Kriterien“ ist. Mit anderen Worten war die Ukraine damit einverstanden, den acquis communautaire ohne jegliche Perspektive auf eine volle EU-Mitgliedschaft zu übernehmen. Immerhin hat die EU zu keiner Zeit angedeutet, dass die freiwillige Harmonisierung zu einer unmittelbaren Anerkennung der ukrainischen Aussicht auf einen Beitritt zur EU führen würde. ${ }^{29}$ Darüber hinaus unterstützt das Programm der Anpassung, höchsten europarechtlichen Traditionen folgend, die Kodifikation des Begriffs "acquis communautaire“. Es berücksichtigt ausführlich die Rechtsprechung des Europäischen Gerichtshofs (EuGH) sowie des Gerichts der Europäischen Union (EuG) als eine formelle Quelle des acquis communautaire, wodurch - wenn auch nur mittelbar - die Anwendung dieser Rechtsprechung in der Ukraine erlaubt wird. Der Erlass des Programms der Anpassung zeigt, dass die ukrainische Regierung zur damaligen Zeit (am Vorabend der

${ }^{28}$ Gesetz der Werchowna Rada der Ukraine „Über das Gesamtstaatliche Programm der Anpassung der Gesetzgebung der Ukraine an die Gesetzgebung der EU“, vom 18. März 2004, Nr. 1629-IV.

${ }^{29}$ Eine Darstellung der Gründe für die Verwehrung eines Beitritts der Ukraine findet sich bei Tiede/Krispenz, Die Ukraine auf dem Weg in die Europäische Union?, Osteuropa-Recht 2008, S. 417-426, $425 \mathrm{f}$. 
„Orangenen Revolution“) das Harmonisierungs-/Anpassungsprogramm als den sinnvollsten Weg erachtete, um von einer der Wellen europäischer Ausdehnung auf Osteuropa erfasst zu werden. Damit kann das Programm der Anpassung als verzweifelter Versuch gewertet werden, die Integration der Ukraine in die EU zu beschleunigen.

Trotz solcher ehrgeizigen „Angleichungsanträge“ seitens der Ukraine haben die Beziehungen zwischen der EU und der Ukraine einen toten Punkt erreicht. Die Annäherung der ukrainischen Rechtsvorschriften an den acquis communautaire schaffte kleine Fortschritte zugunsten der Beziehungen EU-Ukraine. Die Aussicht auf eine volle Mitgliedschaft bestätigt die EU aber bis heute nicht. Vielmehr bestärkt sie den ukrainischen Präsidenten Janukowitsch, von Reden abzusehen, die mit einem möglichen Beitritt der Ukraine zur EU verbunden sind. Stattdessen erwartet man von der Ukraine, dass sie den Verhandlungsprozess über ein neues Assoziationsabkommen abschließt und eine Freihandelszone zwischen ihr und der EU errichtet. ${ }^{30}$

\section{Institutionelle Mechanismen der Rechtsangleichung in der Ukraine}

\section{Gemeinsame Institutionen der EU und der Ukraine}

Die Anpassung ukrainischer Rechtsvorschriften an das Unionsrecht erfolgt innerhalb eines institutionellen Rahmens, der durch das PKA sowie verschiedene nationale Regierungsstellen und Behörden geschaffen wurde. Diese Institutionen weisen die Struktur Europäischer Institutionen auf: Es wurde ein Kooperationsrat eingesetzt, der die Durchführung des PKA überwacht (Art. 85 S. 1 PKA); bei der Erfüllung seiner Aufgaben wird er von einem Kooperationsausschuss unterstützt (Art. 87 Abs. $1 \mathrm{~S}$. 1 PKA); der Gewährleistung eines politischen Dialogs dient der Parlamentarische Kooperationsausschuss (Art. 90 S. 1 PKA). Keinem dieser Organe wurde jedoch die Macht gewährt, rechtsverbindliche Entscheidungen zu treffen und so bedeutenden Einfluss auf den Anpassungsprozess in der Ukraine zu nehmen.

Die wichtigsten politischen Fragen und Handelsstreitigkeiten werden auf „Gipfeltreffen" vom Präsidenten des Rates der Europäischen Union und dem Präsidenten der Kommission einerseits sowie dem Präsidenten des PKA-Landes andererseits entschieden. ${ }^{31}$ Auch in Bezug auf die Ukraine finden entsprechende Treffen statt, obwohl das PKA EU-Ukraine diese gar nicht erwähnt. ${ }^{32}$ Sie spielen eine entschei-

30 Rettman, EU welcomes 'pragmatic' Ukrainian President, EUObserver, 1. März 2010, http://euobserver.com/9/29579, letzter Zugriff am 2. November 2010.

31 Das PKA zwischen der EU und der Russischen Föderation sieht jährlich zwei Treffen dieser Art vor (vgl. dort Art. 7 Abs. 1). An dem Gipfeltreffen nehmen oft andere Europäische Spitzenbeamte teil, wie der den Ratspräsidenten unterstützende Generalsekretär des Rates und Hohe Vertreter für die Gemeinsame Außen- und Sicherheitspolitik (GASP). Siehe gemeinsame Erklärung vom Gipfeltreffen Russland-EU am 29. Mai 2000, Pressemitteilung der EU, Nr. 8976/00 (188).

32 Das fünfte Gipfeltreffen EU-Ukraine fand am 4. Juli 2002 in Kopenhagen statt. Gemeinsame Erklärung von Rasmussen, Präsident des Europäischen Rates, unterstützt vom Generalsekretär des Rates und Hohem Vertreter für die Gemeinsame Außen- und Sicherheitspolitik (GASP), Solana, dem Präsi- 
dende Rolle in der Förderung wirtschaftlicher und politischer Kooperation, einschließlich des Anpassungsprozesses.

Das neue Assoziierungsabkommen zwischen der EU und der Ukraine wird voraussichtlich den allgemeinen, unter dem PKA entwickelten Rahmen beibehalten, obwohl sich mehrere wichtige Unterschiede ergeben werden. Zunächst werden die gemeinsamen Institutionen als "Assoziierungsrat" und "Assoziierungsausschuss" benannt, um den gehobenen Stand des Vertrages widerzuspiegeln. Des Weiteren werden die gemeinsamen Institutionen voraussichtlich die Kompetenz erhalten, verbindliche Entscheidungen $\mathrm{zu}$ erlassen, wie es in den meisten EUAssoziierungsabkommen mit Drittländern der Fall ist. Diese Institutionsreformen sollten zu einer besseren Implementierung und Durchsetzung des neuen Assoziierungsabkommens führen.

\section{Anpassungsprozess innerhalb der ukrainischen Exekutive}

Seit der Einleitung des Programms der Anpassung gab es in der Ukraine bedeutende institutionelle Reformen. Bis 2005 spielte der Präsident der Ukraine die politische Hauptrolle bei der Durchsetzung Europäischer Integrationspolitik im Land. Er lenkte und definierte die Strategie der Integration und setzte die Prioritäten im Bereich der Außenpolitik der Ukraine. Die verfassungsrechtliche Reform der "Orangenen Revolution" im Jahre 2005 sorgte dahingehend für einen Wandel. Der Präsident der Ukraine teilt sich nunmehr viele exekutivrechtliche Kompetenzen zur Ausführung die Integration bezweckender Aufgaben mit dem Ministerkabinett, das seinerseits Behörden, Organisationen, Institutionen und Staatsbeamte ermächtigt. Seit 2005 wurden mehrere Beratungsorgane begründet, um das Ministerkabinett der Ukraine dabei zu unterstützen, die Integrationsstrategie in die EU und andere internationale Institutionen einzufassen. Eines davon ist der Koordinationsrat für die Anpassung der ukrainischen Rechtsvorschriften an das Unionsrecht. ${ }^{33}$ Er umfasst Mitglieder des Ministerkabinetts der Ukraine unter dem Vorsitz des Premierministers. Diese Institution entwirft Jährliche Anpassungspläne, koordiniert den Anpassungsprozess innerhalb der Exekutive, erlässt semi-verbindliche Entscheidungen und kontrolliert die Geschwindigkeit des Integrationsprozesses. Der Koordinationsrat wird unterstützt von zwei mächtigen Exekutivbehörden. Erstere ist das Amt für Europäische Integration im Sekretariat des Ministerkabinetts (Koordinationsamt). Die zweite ist das Department der Anpassung der Gesetzgebung im Justizministerium der Ukraine. Das Koordinationsamt ist gekennzeichnet durch die beträchtliche Unterstützung der ukrainischen Exekutive mittels Koordination, Information sowie Expertise und erfüllt seine Funktion als informelles „Ministerium der Europäischen Integration“. Dennoch ist die Tragweite des Amtes mangels fehlender Kompetenz, verbindliche

dent der Europäischen Kommission, Prodi, und des Präsidenten der Ukraine, Kutschma, Pressemitteilung der EU, Nr. 10607/02 (195). Die Parteien vereinbarten, sich auf die Annäherung der Ukrainischen Gesetzgebung und die Zusammenarbeit auf Gebieten der Energie, des Handels, der Justiz und der Innenpolitik, des Umweltschutzes, des Verkehrs, der Wissenschaft und Technologie zu konzentrieren.

33 Verordnung des Ministerkabinetts „Über den Koordinationsrat für die Anpassung der Ukrainischen Gesetzgebung an die Gesetzgebung der EU“, vom 15. Oktober 2004, Nr. 1365. 
Entscheidungen zu treffen, beschränkt. ${ }^{34}$ Das Department der Anpassung hat den Charakter eines "Think Tank", der dazu berufen ist, die ukrainische Exekutive mit unionsrechtlicher Expertise zu unterstützen und die Vereinbarkeit von Gesetzesentwürfen mit dem Unionsrecht zu überprüfen. Ferner ist es dafür verantwortlich, den acquis communautaire ins Ukrainische zu übersetzen. ${ }^{35}$

Das Ministerkabinett der Ukraine sichert die Implementierung des Programms der Integration in der Praxis. Das hauptsächliche Arbeitspensum wird zwischen den Ministerien, von welchen jedes für einen bestimmten vom Ministerkabinett zugeteilten Bereich verantwortlich ist, aufgeteilt. ${ }^{36}$ Alle Rechtsakte, die vom Ministerkabinett erlassen werden, müssen einem Überwachungs- und Prüfungsverfahren unterzogen werden. Jeder Gesetzesentwurf, der in die vorrangigen Bereiche der Anpassung fällt, muss vom Department der Anpassung auf die Vereinbarkeit mit dem Unionsrecht geprüft werden. Im Falle der Unvereinbarkeit eines vorgelegten Gesetzesentwurfs mit dem Unionsrecht verkündet das Justizministerium Schlussfolgerungen, die Gründe für die Nichtübereinstimmung enthalten können. Dennoch hat das Ministerkabinett - unter Berücksichtigung der entweder zustimmenden oder ablehnenden Schlussfolgerungen des Justizministeriums - das letzte Wort bei der Entscheidung über die Erforderlichkeit, ein bestimmtes Gesetz zu verabschieden. Dies impliziert den weiten Ermessensspielraum des Ministerkabinetts bezüglich der Ausgestaltung der Geschwindigkeit und Tiefe des Anpassungsprozesses in der Ukraine. Allerdings kann angebracht werden, dass die Arbeit des Ministerkabinetts in Bereichen der Harmonisierung/Anpassung ukrainischer Rechtsvorschriften weder von Jurisdiktion oder Legislative noch der Allgemeinheit hinreichend kontrolliert wurde. Das Ministerkabinett ist dem Präsidenten der Ukraine und der Werchowna Rada rechenschaftspflichtig. Dennoch ist es für die Verzögerung in der Harmonisierung/Anpassung nie öffentlich kritisiert worden. Mangelnde Transparenz innerhalb des Ministerkabinetts behinderte ebenfalls die Rechtfertigung seiner Arbeit in der breiten Öffentlichkeit in der Ukraine. Die Jährlichen Anpassungspläne wurden in den Medien nicht angemessen diskutiert und Akademiker sowie Studenten hatten kaum eine Chance, zeitnahe Informationen über neue Initiativen innerhalb des Harmonisierungs-/Anpassungsprozesses zu erhalten.

\section{Anpassungsprozess innerhalb der ukrainischen Legislative}

Bis zum Jahre 2002 wurde der Anpassungsprozess ausschließlich innerhalb der Exekutive unter der Leitung des Präsidenten der Ukraine ausgeübt. Daher gab es weder einen umfassenden rechtlichen noch einen zusammenhängenden institutionellen Mechanismus, um den Anpassungsprozess durch alle Gewalten, einschließlich der Legislative und der Jurisdiktion zu koordinieren. Infolgedessen waren viele

\footnotetext{
${ }^{34}$ Verordnung des Ministerkabinetts „Ü̈ber das Sekretariat des Ministerkabinetts“, vom 31. März 2010, Nr. 286.

35 Verordnung des Ministerkabinetts „Über die Errichtung des Auswärtigen Amts für Angelegenheiten der Anpassung der Gesetzgebung“, vom 24. Dezember 2004, Nr. 1742.

36 Verordnung des Ministerkabinetts „Über die Genehmigung der Geschäftsordnung des Ministerkabinetts", vom 18. Juli 2007, Nr. 950.
} 
der von der Exekutive verabschiedeten ukrainischen Gesetze unvereinbar mit dem primären Recht, das durch die Werchowna Rada (ukrainisches Parlament) erlassen wird. Von 1999 bis 2004 wurden einige Versuche unternommen, alle Gewalten in das kohärente institutionelle Rahmenwerk des Anpassungsprozesses zu fassen. ${ }^{37}$ Der Durchbruch folgte den parlamentarischen Wahlen im Jahre 2002, als die europäischen Ambitionen der Ukraine eine mehrheitliche Bestätigung durch die gewählten politischen Parteien erfuhren. Die Werchowna Rada erkannte die Notwendigkeit ausdrücklich an, Gesetze zu erlassen, die auf die Implementierung des PKA, den Beitritt der Ukraine in die WTO und die Errichtung einer Freihandelszone mit der EU abzielen. Zu diesem Zweck wurde der Parlamentarische Ausschuss für Angelegenheiten der Europäischen Integration gegründet. Dieser ist dafür verantwortlich, Konzepte der Europäischen Integration innerhalb der ukrainischen Legislative voranzubringen und Gesetzesentwürfe, die von der Werchowna Rada verabschiedet werden sollen, auf ihre Vereinbarkeit mit dem Unionsrecht zu überprüfen. Das Verfahrensrecht der Werchowna Rada wurde geändert, um den Erlass von Gesetzen zu vermeiden, die rechtlichen EU-Standards ${ }^{38}$ widersprechen; außerdem wurde das Rahmengesetz Programm der Anpassung im Jahre 2004 erlassen. ${ }^{39}$

\section{Anpassungsprozess innerhalb der ukrainischen Jurisdiktion}

Die ukrainische Jurisdiktion ist am Anpassungsprozess nicht unmittelbar beteiligt. ${ }^{40}$ Ukrainische Gerichtshöfe begegnen allerdings der Notwendigkeit, in ihren Urteilen Unionsrecht anzuwenden. Die Auffassung der ukrainischen Regierung, die freiwillige Harmonisierung nationaler Rechtsvorschriften mit dem Unionsrecht sowie die Übernahme des acquis seien wesentliche Faktoren, um die Integration der Ukraine in die EU zu beschleunigen, hat zu einer positiven Haltung der Gerichtshöfe bezüglich der Berücksichtigung des Unionsrechts in ihren Urteilen geführt. Die Notwendigkeit, Unionsrecht zu berücksichtigen, besteht etwa in Fällen, in denen die Anwendung des PKA in Frage steht. ${ }^{41}$

Aufgrund seiner bedeutenden Stellung in der ukrainischen Rechtsordnung wird das PKA von einigen nationalen Richtern nicht nur als ein verbindliches völkerrechtliches Abkommen wahrgenommen, sondern als etwas mehr. Im Falle der Kollision von PKA-Bestimmungen mit nationalem Recht erkennen die ukrainischen Gerichtshöfe den Vorrang und die unmittelbare Wirkung der PKA-Regelung an. So etwa in

\footnotetext{
${ }^{37}$ Die Strategie der Integration ermächtigte die leitenden, obersten und kommunalen Exekutivbehörden der Ukraine, eng mit der Legislative - der Werchowna Rada - und den relevanten kommunalen Verwaltungsbehörden zusammenzuarbeiten, um die Integration in die EU auf allen Ebenen der ukrainischen Gesellschaft zu gewährleisten.

38 Verordnung der Werchowna Rada der Ukraine „Empfehlungen nach der parlamentarischen Anhörung in Angelegenheiten der Realisierung der Regierungspolitik der Integration der Ukraine in die EU“, vom 17. Januar 2002, Nr. 2999-III.

${ }^{39}$ Gesetz der Werchowna Rada der Ukraine „Über das Gesamtstaatliche Programm der Anpassung der Gesetzgebung der Ukraine an die Gesetzgebung der EU“, vom 18. März 2004, Nr. 1629-IV.

${ }^{40} \mathrm{Zu}$ den Reformbewegungen im ukrainischen Justizsektor siehe Tiede/Krispenz/Schirmer (Fn. 10).

${ }^{41}$ Wie erwähnt stellt das PKA nach der Ukrainischen Verfassung einen Bestandteil der nationalen Rechtsordnung dar und genießt Vorrang gegenüber kollidierenden nationalen Rechtsvorschriften, nicht aber gegenüber der Ukrainischen Verfassung.
} 
der Entscheidung Ryvne Customs Office v. Demyanyk ${ }^{42}$, in der der Oberste Wirtschaftsgerichtshof der Ukraine die unmittelbare Wirkung und den Vorrang des Art. 15 PKA gegenüber dazu in Widerspruch stehenden nationalen Rechtsvorschriften betreffend die nationale Behandlung europäischer Gesellschaften in der Ukraine feststellte. Darüber hinaus rechtfertigen einige ukrainische Gerichtshöfe den Verweis auf das Unionsrecht mit der "Angleichungsklausel“ des Art. 51 PKA. In der Entscheidung Chernomortechflot v. Odessa Tax Office ${ }^{43}$ hatte der Oberste Wirtschaftsgerichtshof der Ukraine sowohl die Transitfreiheit als auch die "Angleichungsklausel“ des Art. 51 PKA als Rechtfertigung dafür herangezogen, dass es der unionsrechtlichen Definition des „internationalen Verkehrskorridors“ gefolgt war.

Die Tragweite der eher vagen Angleichungsverpflichtungen der Ukraine und ihrer weitreichenden Konsequenzen für die ukrainische Rechtsordnung wurde vom Verwaltungsgericht Kiew in der Entscheidung Person v. State Agency in nationalities and religions ${ }^{44}$ hervorgehoben. Darin verwies es auf das ukrainische Gesetz über das "Gesamtstaatliche Programm der Anpassung der Gesetzgebung der Ukraine an die Gesetzgebung der EU“ und stellte fest, dass das Ziel der Anpassung ukrainischer Rechtsvorschriften die Angleichung derselben an den acquis communautaire erfordert, welcher aus dem Primär- und Sekundärrecht sowie den Entscheidungen des EuGH besteht. Dazu nannte das Urteil des Verwaltungsgerichts Kiew neben anderen relevanten Rechtsquellen die Richtlinie 2004/83/EG des Rates vom 29. April $2004^{45}$, die vielfach vom Europäischen Gerichtshof für Menschenrechte (EGMR) als einschlägige Richtlinie angewandt wird.

Die jüngst geschaffene ukrainische Verwaltungsgerichtsbarkeit, die infolge aktiver technischer und fachkundiger Unterstützung internationaler und europäischer Einrichtungen (Europarat, Organisation für Sicherheit und Zusammenarbeit in Europa (OSZE), Europäische Union) ${ }^{46}$ gegründet wurde, hat sich als weiterer Vorkämpfer der Anwendung des aqcuis in ukrainischen Gerichten erwiesen. Hinter der Verwaltungsgerichtsreform in der Ukraine stand die Idee, die Verantwortlichkeit staatlicher Instanzen in Übereinstimmung mit den höchsten Europäischen Grundsätzen zu steigern; bereits andere mittel- und osteuropäische Staaten hatten zuvor eine Verwaltungsgerichtsbarkeit geschaffen, um sich für eine EU-Mitgliedschaft zu qualifizieren. ${ }^{47}$ Dies ermunterte die neu etablierten ukrainischen Verwaltungsgerichte, allgemeine Grundsätze des Unionsrechts zu importieren und sich von Entscheidun-

42 Urteil vom 2. Februar 2005, Nr. 12/267; siehe auch die Entscheidung Closed Stock Company "Chumak" v. Kherson Custom Office, Urteil vom 25. März 2005, Nr. 7/299 und die Entscheidung “Odek" LTD v. Ryvne Custom Office, Urteil vom 22. Februar 2005, Nr. 18/303.

${ }^{43}$ Urteil vom 22. Juni 2004, Nr.28/296-03-6901.

${ }^{44}$ Urteil vom 22. Mai 2008, Nr. 4/48 und Urteil vom 13. Oktober 2008, Nr. 4/375.

${ }^{45}$ Richtlinie 2004/83/EG des Rates vom 29. April 2004 über Mindestnormen für die Anerkennung und den Status von Drittstaatsangehörigen oder Staatenlosen als Flüchtlinge oder als Personen, die anderweitig internationalen Schutz benötigen, und über den Inhalt des zu gewährenden Schutzes, ABl. L 304 vom 30. September 2004, S. 12 - 23.

${ }^{46}$ Alleine die OSZE leitete etwa 25 Workshops für ca. 600 Richter von Verwaltungsgerichten der Ukraine im Zeitraum von 2005 bis 2008 und förderte Austauschprogramme und Schulungen, http://www.osce.org/item/35850.html, letzter Zugriff am 2. November 2010.

47 Emmert, Administrative and Court Reform in Central and Eastern Europe, European Law Journal 2003, S. 288-315. 
gen des EuGH und des EGMR in Fällen der Staatshaftung gegenüber Bürgern inspirieren zu lassen. Grundsätzlich rechtfertigen ukrainische Verwaltungsgerichte die Anwendung von Entscheidungen des EuGH mit der Notwendigkeit, der Rechtsprechung des EGMR zu folgen. So bestimmte das Verwaltungsgericht Kiew in der Entscheidung Tsesarenko v. Representative office of the Pension Fund in Shevchenko district of $K_{i e v}{ }^{48}$, dass der Grundsatz der Rechtsstaatlichkeit in Übereinstimmung mit der Rechtsprechung des EGMR angewandt werden muss. ${ }^{49}$ Das Verwaltungsgericht Kiew begründete seine Entscheidung damit, dass die Implementierung der hohen Europäischen Standards für Menschenrechtsschutz in der Ukraine die Anzahl der Klagen ukrainischer Staatsangehöriger vor dem EGMR gegen die Ukraine reduzieren werde.

In Fällen mit Bezug auf die Staatshaftung gegenüber Bürgern haben die ukrainischen Verwaltungsgerichte einen der ukrainischen Rechtsordnung zuvor unbekannten Grundsatz der Rechtssicherheit entwickelt. So hat in der Entscheidung Person v. Kiev City centre for social assistance ${ }^{50}$ das Verwaltungsgericht Kiew den Grundsatz der Rechtssicherheit aus der Rechtsprechung des EuGH importiert. Es hat bestimmt, dass das Recht von behinderten Menschen, soziale und finanzielle Hilfe vom Staat in Anspruch zu nehmen, aus dem Grundsatz der Rechtssicherheit folgt. Das bedeutet, dass ein Staat die Verletzung seiner Pflicht, Grundrechte zu garantieren, nicht durch das Fehlen spezieller nationaler Rechtsvorschriften rechtfertigen kann. Hierzu berief sich das Verwaltungsgericht Kiew auf die Entscheidung des EuGH im Fall Duyn v. Home Office ${ }^{51}$, die besagt, dass sich die Betroffenen auf staatliche Verpflichtungen berufen können, obwohl diese Verpflichtungen in einem Rechtsetzungsakt niedergelegt sind, der nicht ipso jure in seiner Gesamtheit unmittelbare Wirkung erzeugt. Darüber hinaus entwickelten ukrainische Gerichtshöfe den Grundsatz des Vertrauensschutzes in der Entscheidung Person v. Darnitsa District of Kiev Center for social assistance ${ }^{52}$, in der es um Leistungsansprüche derer geht, die am Einsatz während der Tschernobylkatastrophe beteiligt waren. Das Verwaltungsgericht Kiew folgerte den Grundsatz der Verantwortung des Staates, Betroffenen dieser Katastrophe Entschädigung zu gewähren, aus der Entscheidung Duyn v. Home Office. Insbesondere stellte das Verwaltungsgericht Kiew fest, dass sich der Staat, wenn er seine Verpflichtung, den durch Tschernobyl Betroffenen Entschädigung zu gewähren, formal anerkannt hat, nicht auf sein eigenes Versagen, dieser Verpflichtung gerecht zu werden, berufen kann, um sich seiner Verantwortung zu entziehen. Dies würde ferner einen Verstoß gegen den Grundsatz des Vertrauensschutzes ukrainischer Staatsangehöriger darstellen.

\footnotetext{
${ }^{48}$ Urteil vom 18. November 2008, Nr. 9/556.

${ }^{49}$ Gesetz der Ukraine „Über die Vollstreckung von Beschlüssen und Anwendung der Praxis des Europäischen Gerichtshofs für Menschenrechte“, vom 23. Februar 2006, Nr. 3476-IV.

${ }^{50}$ Urteil vom 25. November 2008, Nr. 2/416; Urteil vom 24. November 2008, Nr. 5/503; Urteil vom 1. Dezember 2008, Nr. 5/451; Urteil vom 10. November 2008, Nr. 5/435.

${ }^{51}$ EuGH, Urteil vom 4. Dezember 1974, Rs. 41/74, Duyn, Slg. 1974, S. 1337, Rn 13/14.

52 Urteil vom 26. Juni 2008, Nr. 4/337.
} 


\section{Schlussbetrachtung}

Der nachhaltige institutionelle Rahmen der Anpassung ukrainischer Rechtsvorschriften an das Unionsrecht befindet sich in einem Stadium der Entwicklung. Als vollkommen ausreichend können die institutionellen Reformen, die bereits stattgefunden haben, dennoch nicht betrachtet werden. In der Ausübung des Anpassungsprozesses bleibt die Werchowna Rada hinter dem Präsidenten der Ukraine und dem Ministerkabinett zurück. Insoweit muss ein ausgewogener Dialog zwischen der Exekutive und der Legislative gesichert werden. Auch darauf ist die weitestgehende Ineffektivität der siebenjährigen Geschichte der Anpassungsreformen in der Ukraine zurückzuführen. Bisher ist kaum eines der ursprünglichen Ziele des Konzeptes der Anpassung umgesetzt worden. Es müssen geeignete Anstrengungen im Bereich Bildung unternommen werden, um das Niveau der europarechtlichen Expertise von Staatsbeamten zu heben. Anderenfalls wird die Durchsetzung des ukrainischen Rechts, das an Unionsrecht angeglichen wurde, dem unvermeidlichen Risiko ausgesetzt, zu scheitern.

Die Ukraine war eine der wenigen ehemaligen sowjetischen Republiken, die sich zu ihren europäischen Ambitionen, die gegenseitige Partnerschaft und Zusammenarbeit $\mathrm{zu}$ verbessern und eine Freihandelszone mit der EU zu errichten, explizit bekannten. Der pro-europäische Kurs der ukrainischen Außenpolitik bleibt in der 20jährigen Geschichte der ukrainischen Unabhängigkeit unverändert. ${ }^{33}$ Die Einleitung des umfassenden Programmes der Anpassung zeigt die Ernsthaftigkeit der europäischen Ambitionen der Ukraine. Tatsächlich ist die Ukraine bereit, ihr nationales Recht an das Unionsrecht anzupassen, das für sie selbst weder bindend ist noch von ihr mitbestimmt werden kann. ${ }^{44}$ Die "freiwillige Harmonisierung" ukrainischer Rechtsvorschriften mit dem Unionsrecht hat alle bedeutenden von der ukrainischen Regierung verabschiedeten rechtlichen Reformen hervorgerufen, seitdem das PKA in Kraft getreten ist. Jedoch ist keine der europäischen Institutionen deutlich geworden bei der Bestimmung des Umfangs des Unionsrechts, das als Modell für die Angleichung betrachtet werden kann. Infolgedessen fand sich die nationale Legislative in einer etwas sonderbaren Situation, als sie sich entscheiden musste zwischen der „blinden“ Übernahme des gesamten acquis und der konsekutiven Angleichung von ukrainischem Recht an das primäre und sekundäre Unionsrecht wie sie etwa durch die vorrangigen Bereiche des PKA EU-Ukraine und der Assoziierungsagenda EU-Ukraine definiert wurde.

In Anbetracht des Problems, verschiedene Modelle der Angleichung in Übereinstimmung zu bringen, hat die Regierung der Ukraine die „Angleichung“ der Rechts-

\footnotetext{
${ }^{53}$ Der Präsident der Ukraine Janukowitsch wiederholte öffentlich sein Ziel, die ukrainische Außenpolitik auf eine volle Mitgliedschaft in der EU zu richten, http://www.president.gov.ua/news/17314.html, letzter Zugriff am 2. November 2010.

${ }^{54}$ Dieser Prozess wurde von Evans als „voluntary harmonisation“ bezeichnet (Evans, Voluntary Harmonisation in Integration between the European Community and Eastern Europe, European Law Review 1997, S. 201 - 220). Andere Autoren definieren diesen Prozess als „autonomous adaptation ", siehe nur Müller-Graff, The Legal Framework for the Enlargement of the Internal Market in Central and Eastern Europe, Maastricht Journal of European and Comparative Law 1999, S. 192 - 207, 196.
} 
vorschriften durch den ebenso vagen Begriff der "Anpassung“ ersetzt. Art. 1 der Strategie der Integration stellt ausdrücklich fest, dass „die Anpassung der ukrainischen Rechtsvorschriften an Gemeinschaftsrecht von der Angleichung an das gegenwärtige Europäische Rechtssystem umfasst ist". An sich sieht die Strategie der Integration die „Reform der ukrainischen Rechtsordnung in Übereinstimmung mit europarechtlichen Standards" in den in Art. 51 PKA bestimmten Bereichen vor, soweit wirtschaftliche, politische und soziale Folgen einer entsprechenden Anpassung für die Ukraine angemessen sind..$^{55}$ Daher stattet die "Anpassung" der ukrainischen Rechtsvorschriften an das Unionsrecht die ukrainische Regierung einerseits mit dem Ermessen aus, den Prozess der Anpassung in Übereinstimmung mit nationalen Interessen $\mathrm{zu}$ steuern. Andererseits verlangsamt sie aber die Entwicklungsgeschwindigkeit der Angleichungsreformen in der Ukraine.

Die Komplexität des Anpassungsprozesses ist durch die Doppeldeutigkeit der EUPolitik bezüglich der Ukraine quasi „vorprogrammiert“. Die „Angleichungsklauseln" im zukünftigen Assoziierungsabkommen der EU mit der Ukraine müssen präzisier sein und den Umfang des von der Ukraine anzunehmenden Unionsrechts im Detail klären. Der Erfolg rechtlicher Reformen in der Ukraine erfordert eine kohärente und konsequente Leitlinie seitens der EU hinsichtlich zu setzender Prioritäten und der Methodik des Angleichungsprozesses.

55 Gemeinsame Strategie des Europäischen Rates vom 11. Dezember 1999 für die Ukraine (1999/877/GASP), ABl. L 331 vom 23. Dezember 1999, S. 1 - 10. Der Europäische Rat von Helsinki betonte, dass die EU einer engen Zusammenarbeit mit der Ukraine im Rahmen des Europarates und der OSZE besondere Bedeutung beimisst und in diesem Zusammenhang die Ukraine auffordert, ihren Verpflichtungen nachzukommen und ihre Rechtsvorschriften so anzupassen, dass sie den Regeln und Normen des Europarates entsprechen und dass insbesondere die Verpflichtungen erfüllt werden, die die Ukraine bei ihrem Beitritt zum Europarat im Jahr 1995 eingegangen ist. 